\title{
Antibacterial Activity of Yogurt Cheese Made from Barki Sheep Milk Supplemented with Olive Oil
}

\author{
Mervat M. EL-Bayoumi
}

\section{ABSTRACT}

The purpose of this study was investigating the antibacterial effects of olive oil supplemented to cheese yoghurt made from Barki sheep milk, on the growth of some probiotic bacterial strains (Bifidobacterium bifidum (ATCC15708), Lactobacillus acidophilus (ATCC4356), Lactobacillus delbrueckii spp. bulgaricus (ATCC7995) and Streptococcus thermophilus (DSM20259) as well as some pathogenic bacterial strains. Results showed that olive oil $(1 \%)$ had no effect on the growth of all probiotic bacterial strains used in Barki cheese yoghurt making. No yeasts, moulds, Enterobacteria spp and Staphylococcus spp were detected in cheese yoghurt containing olive oil (1\%) through the entire storage period (21days). However, control treatment had Yeast and Moulds at the end of storage period. When four strains of pathogens were added to cheese yoghurt containing different probiotic bacteria and supplemented with olive oil $(1 \%)$ then stored at $5 \mathrm{C}$ for 72 hours, results revealed that $E$. coil (ACCT8739) was the most sensitive microorganism while, Staphylococcus aureus (ATCC6538) was the most resistant one. The antibacterial activity of cheese yoghurt supplemented with olive oil ( $1 \%$ ) was higher than control treatment because olive oil supported the growth of Lactic acid bacteria. From these results, it is recommended that olive oil (1\%) can be used as a natural and safe antimicrobial substance in Barki cheese yoghurt and other dairy products, and olive oil may well have the beneficial role in promoting probiotic bacteria and inhibiting harmful bacteria.

Submitted : July 22, 2021

Published : August 31, 2021

ISSN: $2684-1827$

DOI: 10.24018 /ejfood.2021.3.4.344

\section{Mervat M. EL-Bayoumi*}

Breeding of Animal and Poultry Department, Desert Research Center, Egypt.

(e-mail: Merfat13373@gmail.com)

*Corresponding Author

Keywords: antibacterial activity, Barki sheep milk, Barki cheese yoghurt, olive oil, pathogenic bacteria, probiotics.

\section{INTRODUCTION}

Barki sheep belongs to Barbary breed which inhabits the hot area of North Africa [1]. Smaller fat globule diameter and greater percentage of short chain fatty acids contribute to easier and more rapid digestion of sheep milk. Short-chain fatty acids such as caproic, caprylic and capric give sheep milk the special taste and aroma and have health benefits [2]. Sheep milk is rich in vitamins A, B and E, calcium, phosphorous, potassium and magnesium than cow's milk [3]. Meanwhile, it is a unique product with high nutritional qualities containing shorter - chain fatty acid, more protein, more calcium, and more vitamins than cow's milk. Olive oil is classified as virgin olive oil if it has been extracted exclusively by mechanical or physical procedures such as milling, beating, centrifugation and decantation [4]. The importance of virgin olive oil is related to its high levels of mono-unsaturated fatty acids (mainly oleic acid) and to the presence of minor components including aliphatic and triterpenic alcohols, sterols, hydrocarbons, volatile compounds, and several antioxidants [5]. Limited studies have been carried out on the utilization of olive oil in dairy products. Concentrated yoghurt or cheese yoghurt is popularly known as Labneh in the Middle East, as strained yoghurt in Greece, the rest of Europe and as Suzme yoghurt in Turkey [6], [7].
In the last decades, consumer in the field of food production have considerably changed; consumers believe that foods contribute directly to their health [8] such as olive oil is needed. On the other hand, several researches were done to improve the quality and shelf-life of Labneh by different attitudes such as using olive oil [9], [10] and by using essential oil [11]-[15]. Probiotic bacteria are recognized for their fermentative ability and thus enhancing food safety, improving organoleptic attributes, enriching nutrients, and increasing health benefits [16]-[19]. It is well known that milk fermentation using lactic acid bacteria results in increase its shelf-life as interest to develop a variety of fermented milk products for other beneficial purpose, particularly for health purpose and preventing of toxins produced by food borne pathogens and spoilage bacteria enter human body [16], [18], [20]-[26]. Therefore, the objective of this study was investigating the antibacterial effects of some probiotic bacterial strains as well as olive oil on the activity of some food borne pathogens and spoilage bacteria.

\section{MATERIAL AND METHODS}

\section{A. Milk Samples}

Barki sheep milk samples were obtained from Maryoot Research Station, Al-Amaryia, Alexandria, Egypt. 


\section{B. Bacterial Strains}

All bacterial strains include Bifidobacterium bifidum (ATCC15708), Lactobacillus acidophilus (ATCC4356), Lactobacillus delbrueckii ssp bulgaricus (ATCC7995), Streptococcus thermophilus (A) as well as Escherichia coli (ACCT8739), Staphylococcus aureus (ATCC6538), Salmonella typhimurim (ACCT25566) and Bacillus cereus (ATCC9639) were obtained from MIRCEN Center, Faculty of Agriculture, Ain Shams University, Egypt.

Virgen olive oil obtained from El-Amryia local market, Alexandria, Egypt.

\section{Preparation of Probiotic Barki Cheese Yoghurt (Labneh)}

Barki sheep milk (1.5\% fat) was divided into two portions. The first portion had no olive oil, while the second one supplemented with $1 \%$ olive oil. The two portions were heated at $90{ }^{\circ} \mathrm{C} / 10 \mathrm{~min}$, cooled to $42{ }^{\circ} \mathrm{C}$ and then subdivided into four equal portions which inoculated with Bifidobacterium bifidium (ATCC15708), L. acidophilus (ATCC4356), Lactobacillus delbrueckii ssp bulgaricus (ATCC35009) and Streptococcus thermophiles (DSM20259), respectively. All portions with olive oil treatments were inoculated with four strains of pathogenic bacterial cultures as Escherichia coli (ACCT8739) (portion 1), Staphylococcus aureus (ATCC6538) (portion 2), Bacillus cereus (ATCC9639) (portion 3) and Salmonella typhimurium (ACCT25566) (portion 4). Then all portions were incubated at $42{ }^{\circ} \mathrm{C}$ for 24 hours. After fermentation. The coagulum was placed in a hanging cloth bag to drain the whey [9]. Labneh samples of all treatments were analysed at 1, 7, 14 and 21 days of storage for $\mathrm{PH}$, viable cell counts of probiotic bacteria, pathogenic bacteria, and antibacterial activity.

\section{Microbial Growth and Enumeration Microorganisms}

Samples ( $10 \mathrm{~g})$ of contaminated Barki cheese yoghurt were taken after $0,12,24,36,48,60$ and 72 hours while, control samples after 1, 7, 14, and 21 days. Then mixed with $90 \mathrm{ml}$ of $0.1 \%$ sterilized peptone water and then homogenized for 5 min with lab blender (MX32). Homogenized samples were serially diluted in peptone solution and plated for bacterial enumeration according to pour plate method. $1 \mathrm{ml}$ of the serial diluted samples were inoculated into molten MRS media, M17 media, MacConkey media, Mannitol salt agar media, Nutrient agar media, Sabouraud dextrose agar media for Bifidobacterium bifidium, L. acidophilus, L. bulgaricus, Enterobacteria spp, Staphylococcus spp count, Bacillus spp count and Yeast and Moulds count, respectively. Plates were incubated at $37{ }^{\circ} \mathrm{C}$ for 48 hours. Then CFUs of the microbes were counted on plates. The experiments were performed in quadruplicates and then the average of four parallel measurements of count in CFU/ml was reported.

\section{E. Antibacterial Activity Measurement}

Antibacterial activity of supernatant of yoghurt cheese supplemented with olive oil against Escherichia coli (ACCT8739), Staphylococcus aureus (ATCC6538), Bacillus cereus (ATCC9639) and Salmonella typhimurium (ACCT25566) was carried out at 1, 7, 14 and 21 days of storage at $5{ }^{\circ} \mathrm{C}$. The experiments were performed in quadruplicates and the average of the four parallel and measurements of inhibition zone in $\mathrm{cm}$ was reported.

\section{RESUlTS AND DisCUSSION}

Table I shows the PH values of different probiotic Barki cheese yoghurt supplemented with olive oil $(1 \%)$ at $1,7,14$ and 21 days of storage at $5{ }^{\circ} \mathrm{C}$. The $\mathrm{PH}$ values of both control and olive oil cheese yoghurt samples were decreased with time progress, however, no significant difference between both treatments.

TABLE I: CHANGES IN PH VALUES OF B ARKI SHEEP CHEESE YOGHURT MADE USING DIFFERENT PROBIOTIC STRAINS AND SUPPLEMENTED WITH OLIVE OIL AND STORED FOR 21 DAYS AT $5^{\circ} \mathrm{C}$

\begin{tabular}{|c|c|c|c|c|c|c|}
\hline \multirow{2}{*}{ Probiotic Strain } & \multirow{2}{*}{$\begin{array}{l}\text { Olive } \\
\text { oil \% }\end{array}$} & \multirow{2}{*}{$\begin{array}{c}\text { Initial } \\
\mathrm{PH}\end{array}$} & \multicolumn{4}{|c|}{ Storage Dayes } \\
\hline & & & 1 & 7 & 14 & 21 \\
\hline \multicolumn{7}{|l|}{ Bifidobacterium } \\
\hline bifidum & 0.0 & 6.62 & 5.42 & 5.35 & 5.30 & 5.19 \\
\hline (ATCC15708) & 1.0 & 6.62 & 5.45 & 5.38 & 5.32 & 5.30 \\
\hline L. acidophilus & 0.0 & 6.61 & 5.46 & 5.34 & 5.30 & 5.30 \\
\hline (ATCC4356) & 1.0 & 6.61 & 5.48 & 5.43 & 5.44 & 5.26 \\
\hline Strep thermophilus & 0.0 & 6.60 & 5.54 & 5.47 & 5.30 & 5.12 \\
\hline (DSM20259) & 1.0 & 6.60 & 5.57 & 5.45 & 5.33 & 5.18 \\
\hline \multirow{2}{*}{$\begin{array}{l}\text { L. bulgaricus } \\
\text { (ATCC7995) }\end{array}$} & 0.0 & 6.62 & 5.55 & 5.44 & 5.35 & 5.12 \\
\hline & 1.0 & 6.62 & 5.58 & 5.48 & 5.40 & 5.18 \\
\hline
\end{tabular}

Table II shows the growth rates of probiotic bacteria in Barki cheese yoghurt supplemented with olive oil (1\%) at 1 , 7,14 and 21 days of storage at $5{ }^{\circ} \mathrm{C}$. In all control samples the viable cell count of Bifidobacterium bifidium (ATCC15708), Lactobacillus acidophilus (ATCC4356), Lactobacillus delbrueckii ssp bulgaricus (ATCC35009) and Streptococcus thermophiles (DSM20259) were increased gradually up to $14^{\text {th }}$ day of storage and then decreased. However, in olive oil $(1 \%)$ treatment, it was noticed that the activity of all probiotic strains was at highest level at $7^{\text {th }}$ day of storage then decreased. Generally, no significant difference between control and olive oil treatment in viable count of all probiotic. This mean that olive oil had no effect of the activity of all probiotic bacterial strains. Thus, it can be proposed that the addition of olive oil $(1 \%)$ to all studied probiotic bacterial strains is a suitable combination, and sufficient to exert the health benefits of the host. These results were agreement to [27]. The minimum required level of probiotic bacteria to be useful for the consumer 's body is $10^{7} \mathrm{cfu} / \mathrm{ml}$ of living bacteria and the level in the present study was found to be $10^{10}$, thus, it could be beneficial for the consumers [27].

It was reported that combined either aqueous or alcoholic olive leaf extracts with skim milk containing Bifidobacterium infants and Lactobacillus acidophilus increased viability of both probiotic strains were observed after $16 \mathrm{~h}$ incubation period for olive extract concentration below $3.0 \mathrm{mg}$ Catechin Equivalents $/ \mathrm{ml}$, whereas adding $5.0 \mathrm{mg}$ Catechin Equivalents $/ \mathrm{ml}$ resulted in significantly decreased probiotic viability [28]. The concentration at which olive oil inhibited microbial growth was greater than a previous study where 2 $\mathrm{ml}$ of olive oil exerted bactericidal activity against probiotic microorganisms such as L. acidophilus and Bifidobacterium bifidum when placed in bactericidal activity assay [29]. In other study, mixed cinnamon or licorice with probiotic yoghurt containing Lactobacillus acidophilus LA-5 and NCMF, B. lactis BB-12, and Lactobacillus casi LC-10 were 
evaluated [30]. It was found that both the addition of cinnamon or licorice increased antioxidant activity in the probiotic yoghurt and correspondingly, viability of Lactobacillus species was reduced during fermentation and storage. A small amount of red ginseng extract promoted the growth of Lactic acid bacteria; however, a large amount inhibited the growth of them [31]. In a study on the effects of soya powder on the growth of Lactobacillus acidophilus and Bifidobacterium bifidum, in probiotic products, it was demonstrated that the shelf life for the acidity reaching the desired level during incubation decreased for the milk with both bacteria and combined soya and malt, compared to the milk with only soya [27], [32].

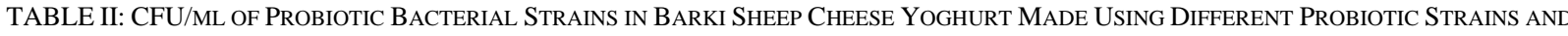
SUPPLEMENTED WITH OLIVE OIL AND STORED FOR 21 DAYS AT $5{ }^{\circ} \mathrm{C}$

\begin{tabular}{|c|c|c|c|c|c|}
\hline \multirow{2}{*}{ Probiotic Strain } & \multirow{2}{*}{ Olive oil \% } & \multicolumn{4}{|c|}{ Storage Days } \\
\hline & & 1 & 7 & 14 & 21 \\
\hline Bifidobacterium & 0.0 & $32.0 \times 10^{10}$ & $43.2 \times 10^{10}$ & $45.1 \times 10^{10}$ & $29.5 \times 10^{10}$ \\
\hline bifidum (ATCC15708) & 1.0 & $12.55 \times 10^{10}$ & $22.32 \times 10^{10}$ & $20.0 \times 10^{10}$ & $9.25 \times 10^{10}$ \\
\hline L. acidophilus & 0.0 & $30.0 \times 10^{10}$ & $40.28 \times 10^{10}$ & $42.32 \times 10^{10}$ & $38.21 \times 10^{10}$ \\
\hline (ATCC4356) & 1.0 & $13.22 \times 10^{10}$ & $15.30 \times 10^{10}$ & $8.32 \times 10^{10}$ & $5.32 \times 10^{10}$ \\
\hline Strep thermophilus & 0.0 & $40.3 \times 10^{10}$ & $49.2 \times 10^{10}$ & $58.1 \times 10^{10}$ & $53.17 \times 10^{10}$ \\
\hline (DSM20259) & 1.0 & $15.20 \times 10^{10}$ & $26.4 \times 10^{10}$ & $22.0 \times 10^{10}$ & $18.1 \times 10^{10}$ \\
\hline L. bulgaricus & 0.0 & $35.0 \times 10^{10}$ & $40.20 \times 10^{10}$ & $50.33 \times 10^{10}$ & $46.11 \times 10^{10}$ \\
\hline (ATCC7995) & 1.0 & $14.53 \times 10^{10}$ & $23.11 \times 10^{10}$ & $20.0 \times 10^{10}$ & $16.22 \times 10^{10}$ \\
\hline
\end{tabular}

It was demonstrated that Vanillin promoted the growth of the bacteria in probiotic milk and yoghurt [33]. Taking into account the results of the above- mentioned studies investigating the effects of malt, soya, ginseng and dill on the growth of L. acidophilus and B. bifidum, we can conclude that they all enhance the bacterial growth in dairy products. Results revealed also that no Enterobacteria spp or Staphylococcus spp were present in control or olive oil treatment. Yeast and molds were also not detected through storage in olive oil treatment, however control treatment had Yeast and Moulds at the end of storage period $21^{\text {st }}$ day. These results are in accordance with that reported by EL-Sayed et al. [9] and Thabet et. al. [13].

Tables (III-VI) show zone inhibition assay of supernatant of uncontaminated Barki cheese yoghurt supplemented with olive oil $1 \%$ containing different probiotic bacteria against pathogenic bacteria stored for $1,7,14$ and 21 days at $5{ }^{\circ} \mathrm{C}$. Results showed that the most sensitive microorganism was $E$. coli (ACCT8739), followed by Salmonella typhimurium (ACCT25566) then Bacillus cereus (ATCC9639) and the most resistant one was Staphylococcus aureus (ATCC6538). Streptococcus thermophilus (DSM20259) supernatant had the highest inhibition zone against E. coli (CCT8739), Salmonella typhimurium (ATCC25566) and Bacillus cereus (ATCC9639). In olive oil $1 \%$ treatment, Bifidobacterium bifidum (ATCC15708) have the highest inhibition zone against Staphylococcus aureus (ATCC6538). Our results are similar to that reported by Eduardo et al. [34], they stated that olive oil had a strong bactericidal action against abroad spectrum of microorganisms, this effect being higher in general against Gram-positive than Gram -negative bacteria. Cornu et al. [35] studied inhibiting effect of phenolic compounds on probiotic bacteria, according to their constitution and concentration and the bacterial strain. The growth rate stimulation by phenolic compounds and the increase in cell incubation could be related to their ability to metabolize these phenolic compounds [36]-[38]. Olive oil had fatty acids that are responsible for the bactericidal action. Although it has been reported that fatty acids possess antimicrobial activity against bacteria and Yeasts [39] and Alfa-Beta - unsaturated aldehydes from olives and olive oil flavor have also been demonstrated to possess antibacterial activity against pathogens of human intestinal and respiratory tracts [40], [41].

TABLE III: ANTIBACTERIAL ACTIVITY OF PROBIOTIC SUPERNATANT OF YoghuRT CHEESE SuPPLEMENTED WITH OLIVE OIL AGAINST ESCHERICHIA

\begin{tabular}{|c|c|c|c|c|c|c|c|c|}
\hline & & & $L I$ & $T 87$ & & & & \\
\hline Storage & $\begin{array}{r}\text { Bifid } \\
b \\
(A T \\
\end{array}$ & $\begin{array}{l}\text { erium } \\
n \\
708)\end{array}$ & $\begin{array}{l}\text { acidc } \\
(\text { ATC }\end{array}$ & $\begin{array}{l}\text { hilus } \\
\text { 4356) }\end{array}$ & bul & $\begin{array}{l}\text { ricus } \\
7995)\end{array}$ & $\begin{array}{l}\text { ther } \\
\text { (DS }\end{array}$ & $\begin{array}{l}\text { ep } \\
\text { philus } \\
\text { 0259) }\end{array}$ \\
\hline (days) & & & & Olive & $1 \%$ & & & \\
\hline & 0.0 & 1.0 & 0.0 & 1.0 & 0.0 & 1.0 & 0.0 & 1.0 \\
\hline & & & Zone $\mathrm{i}$ & ibitio & assay & $\mathrm{cm})$ & & \\
\hline 1 & 0.6 & 1.0 & 0.7 & 0.9 & 1.0 & 0.9 & 1.0 & 1.0 \\
\hline 7 & 1.3 & 2.0 & 1.3 & 1.9 & 1.7 & 2.0 & 1.9 & 2.4 \\
\hline 14 & 1.2 & 1.9 & 1.3 & 1.6 & 1.6 & 2.0 & 1.9 & 2.4 \\
\hline 21 & 1.0 & 1.8 & 1.0 & 1.6 & 1.6 & 1.9 & 1.7 & 2.2 \\
\hline
\end{tabular}

TABLE IV: ANTIBACTERIAL ACTIVITY OF PROBIOTIC SUPERNATANT OF YogHURT CHEESE SUPPLEMENTED WITH OLIVE OIL AGAINST BACILLUS CEREUS (ATCC6538)

\begin{tabular}{|c|c|c|c|c|c|c|c|c|}
\hline \multirow{4}{*}{$\begin{array}{l}\text { Storage } \\
\text { (days) }\end{array}$} & \multicolumn{2}{|c|}{$\begin{array}{c}\text { Bifidobacterium } \\
\text { bifidum } \\
\text { (ATCC15708) }\end{array}$} & \multicolumn{2}{|c|}{$\begin{array}{c}\text { L. } \\
\text { acidophilus } \\
\text { (ATCC4356) }\end{array}$} & \multicolumn{2}{|c|}{$\begin{array}{c}\text { L. } \\
\text { bulgaricus } \\
\text { (ATCC7995) }\end{array}$} & \multicolumn{2}{|c|}{$\begin{array}{c}\text { Strep } \\
\text { thermophilus } \\
\text { (DSM20259) }\end{array}$} \\
\hline & \multicolumn{8}{|c|}{ Olive oil \% } \\
\hline & 0.0 & 1.0 & 0.0 & 1.0 & 0.0 & 1.0 & 0.0 & 1.0 \\
\hline & \multicolumn{8}{|c|}{ Zone inhibition assay $*(\mathrm{~cm})$} \\
\hline 1 & 1.0 & 1.9 & 0.8 & 0.9 & 0.8 & 1.0 & 0.9 & 1.2 \\
\hline 7 & 2.1 & 2.6 & 1.2 & 2.0 & 1.3 & 2.1 & 1.4 & 2.1 \\
\hline 14 & 2.0 & 2.5 & 1.2 & 2.0 & 1.2 & 2.1 & 1.3 & 2.1 \\
\hline 21 & 2.0 & 2.4 & 1.0 & 1.9 & 1.0 & 2.0 & 1.1 & 2.0 \\
\hline
\end{tabular}

TABLE V: ANTIBACTERIAL ACTIVITy OF PROBIOTIC SUPERNATANT OF YOGHURT CHEESE SUPPLEMENTED WITH OLIVE OIL AGAINST STAPHYLOCOCCUS AUREUS (ATCC6538)

\begin{tabular}{|c|c|c|c|c|c|c|c|c|}
\hline \multirow{4}{*}{$\begin{array}{c}\text { Storage } \\
\text { (days) }\end{array}$} & \multicolumn{2}{|c|}{$\begin{array}{c}\text { Bifidobacterium } \\
\text { bifidum } \\
\text { (ATCC15708) }\end{array}$} & \multicolumn{2}{|c|}{$\begin{array}{c}\text { L. } \\
\text { acidophilus } \\
(\text { ATCC4356) }\end{array}$} & \multicolumn{2}{|c|}{$\begin{array}{c}\text { L. } \\
\text { bulgaricus } \\
\text { (ATCC7995) }\end{array}$} & \multicolumn{2}{|c|}{$\begin{array}{c}\text { Strep } \\
\text { thermophilus } \\
\text { (DSM20259) }\end{array}$} \\
\hline & \multicolumn{8}{|c|}{ Olive oil \% } \\
\hline & 0.0 & 1.0 & 0.0 & 1.0 & 0.0 & 1.0 & 0.0 & 1.0 \\
\hline & \multicolumn{8}{|c|}{ Zone inhibition assay $*(\mathrm{~cm})$} \\
\hline 1 & 0.8 & 1.0 & 0.7 & 1.0 & 0.8 & 1.0 & 0.9 & 1.2 \\
\hline 7 & 1.1 & 2.2 & 1.2 & 2.1 & 1.2 & 2.2 & 1.3 & 2.3 \\
\hline 14 & 1.0 & 2.2 & 1.1 & 2.0 & 1.2 & 2.2 & 1.2 & 2.2 \\
\hline 21 & 1.0 & 2.0 & 1.0 & 2.0 & 1.0 & 2.2 & 1.1 & 2.1 \\
\hline
\end{tabular}


TABLE VI: ANTIBACTERIAL ACTIVITY OF PROBIOTIC SUPERNATANT OF YOGHURT CHEESE SUPPLEMENTED WITH OLIVE OIL AGAINST SALMONELLA TYPHIMURIUM (ATCC25566)

\begin{tabular}{|c|c|c|c|c|c|c|c|c|}
\hline \multirow{4}{*}{$\begin{array}{c}\text { Storage } \\
\text { (days) }\end{array}$} & \multicolumn{2}{|c|}{$\begin{array}{c}\text { Bifidobacterium } \\
\text { bifidum } \\
\text { (ATCC15708) }\end{array}$} & \multicolumn{2}{|c|}{$\begin{array}{c}\text { L. } \\
\text { acidophilus } \\
\text { (ATCC4356) }\end{array}$} & \multicolumn{2}{|c|}{$\begin{array}{c}\text { L. } \\
\text { bulgaricus } \\
(\text { ATCC7995) }\end{array}$} & \multicolumn{2}{|c|}{$\begin{array}{c}\text { Strep } \\
\text { thermophilus } \\
\text { (DSM20259) }\end{array}$} \\
\hline & \multicolumn{8}{|c|}{ Olive oil \% } \\
\hline & 0.0 & 1.0 & 0.0 & 1.0 & 0.0 & 1.0 & 0.0 & 1.0 \\
\hline & \multicolumn{8}{|c|}{ Zone inhibition assay $*(\mathrm{~cm})$} \\
\hline 1 & 0.9 & 1.0 & 0.8 & 1.0 & 1.0 & 1.4 & 1.0 & 1.5 \\
\hline 7 & 1.2 & 2.2 & 1.2 & 1.4 & 1.4 & 2.4 & 1.6 & 2.6 \\
\hline 14 & 1.2 & 2.1 & 1.1 & 2.1 & 1.3 & 2.3 & 1.6 & 2.5 \\
\hline 21 & 1.1 & 2.1 & 1.0 & 2.1 & 1.2 & 2.3 & 1.5 & 2.4 \\
\hline
\end{tabular}

*The experiments were performed in quadruplicates and then the average of the four parallel measurements of the inhibition zone in $\mathrm{cm}$ were reported.

Tables (VII-X) show the antibacterial activity of contaminated Barki cheese yoghurt supplemented with olive oil $1 \%$ at storage time $0,12,24,36,48,60$ and 72 hours at $5{ }^{\circ} \mathrm{C}$ against E. coli (ACCT8739), Salmonella typhimurium (ACCT25566), Bacillus cereus (ATCC9639) and Staphylococcus aureus (ATCC6538). The results showed various degrees of inhibition against the four bacterial strains. In olive oil $1 \%$ treatment the growth of E. coil (ACCT8739) and Salmonella typhimurium (ATCC25566) was stopped in the presence of Streptococcus thermophiles (DSM20259), and L. bulgaricus (ATCC7995) after $24 \mathrm{~h}$. However, the growth of Bacillus cereus (ATCC9639) was stopped with all different probiotic bacteria after $36 \mathrm{~h}$ Bifidobacterium bifidum (ATCC15708) was stopped the growth of
Staphylococcus aureus (ATCC6538) after $24 \mathrm{~h}$

Markrin et al. [42] reported that olive leaf extract was able to inhibit the growth of $C$. albicans after 1day of contact. It must be assumed that active compounds of the olive leaf extract, mainly phenolic compounds, which are different from olive oil. Olive leaf extract was more inhibitory against $E$. coli than against $C$. albicans. It has been reported that virgin olive oil was effective in preserving yoghurt cheese inoculated with the Kluyveromyces marxianus [43]. Phenolic compounds from berry extracts inhibited the growth of Gram -negative [44]. Olive oil exhibited bactericidal activity against $E$. coli and $C$. perfringens, which grow in the intestine. Park et al. [45] reported that many plant extracts and foods such as tea may improve the intestinal microbial by inhibiting harmful microorganisms but also promoting or maintaining the beneficial ones such as lactic acid bacteria [46]. The bio active compounds such as poly phenols can be absorbed before they reach the colon [47] and they are even transformed by intestinal bacteria [48] olive oil. Pretty et al. [49] reported that olive oil can decrease both bacterial growth and adhesion. Eduardo et al. [34] found that olive oil has a very strong bactericidal effect against $S$. mutans. Raford et al. [50] observed a faster death rate of $S$. enterica in mayonnaise made with olive oil. Alwaili [51] found that honey, olive oil and bee wax have been used to treat the disease diaper dermatitis.

TABLE VII: CFU/ML OF ESCHERICHIA COLI (ATCC8739) INOCULATED IN BARKI SHEEP CHEESE YOGHURT MADE USING DIFFERENT

\begin{tabular}{|c|c|c|c|c|c|c|c|c|}
\hline \multirow{3}{*}{$\begin{array}{l}\text { Storage } \\
\text { (h) }\end{array}$} & \multicolumn{2}{|c|}{$\begin{array}{c}\text { Bifidobacterium bifidum } \\
\text { (ATCC15708) }\end{array}$} & \multicolumn{2}{|c|}{$\begin{array}{l}\text { L. acidophilus } \\
\text { (ATCC4356) }\end{array}$} & \multicolumn{2}{|c|}{$\begin{array}{l}\text { L. bulgaricus } \\
\text { (ATCC7995) }\end{array}$} & \multicolumn{2}{|c|}{$\begin{array}{l}\text { Strep thermophilus } \\
\text { (DSM20259) }\end{array}$} \\
\hline & \multicolumn{8}{|c|}{ Olive oil \% } \\
\hline & 0.0 & 1.0 & 0.0 & 1.0 & 0.0 & 1.0 & 0.0 & 1.0 \\
\hline 0 & $8.22 \times 10^{7}$ & $9.2 \times 10^{7}$ & $7.7 \times 10^{7}$ & $8.8 \times 10^{8}$ & $8.4 \times 10^{7}$ & $8.9 \times 10^{7}$ & $8.44 \times 10^{7}$ & $10.0 \times 10^{8}$ \\
\hline 12 & $3.0 \times 10^{5}$ & $2.0 \times 10^{8}$ & $6.0 \times 10^{6}$ & $5.3 \times 10^{6}$ & $3.3 \times 10^{6}$ & $8.0 \times 10^{5}$ & $10.0 \times 10^{7}$ & $5.3 \times 10^{4}$ \\
\hline 24 & $5.0 \times 10^{3}$ & $3.3 \times 10^{4}$ & $5.0 \times 10^{5}$ & $3.5 \times 10^{8}$ & $5.2 \times 10^{4}$ & & $7.0 \times 10^{6}$ & \\
\hline 36 & $6.0 \times 10^{2}$ & & $9.0 \times 10^{3}$ & & $6.8 \times 10^{2}$ & & $5.0 \times 10^{2}$ & \\
\hline 48 & & & & & & - & & \\
\hline 60 & & & & & 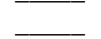 & & & \\
\hline 72 & & & & & & & & \\
\hline
\end{tabular}

TABLE VIII: CFU/ML OF STAPHYLOCOCCUS AUREUS (ATCC6538) INOCULATED IN BARKI SHEEP CHEESE YOGHURT MADE USING DIFFERENT PROBIOTIC STRAINS AND SUPPLEMENTED WITH OLIVE OIL AND STORED FOR $72 \mathrm{H}$ AT $5^{\circ} \mathrm{C}$

\begin{tabular}{|c|c|c|c|c|c|c|c|c|}
\hline \multirow[t]{3}{*}{$\begin{array}{l}\text { Storage } \\
\text { (hrs) }\end{array}$} & \multicolumn{2}{|c|}{$\begin{array}{l}\text { Bifidobacterium } \\
\text { (ATCC15708) }\end{array}$} & \multicolumn{2}{|c|}{$\begin{array}{l}\text { L. acidophilus } \\
\text { (ATCC4356) }\end{array}$} & \multicolumn{2}{|c|}{$\begin{array}{l}\text { L. bulgaricus } \\
(\text { ATCC7995) }\end{array}$} & \multicolumn{2}{|c|}{$\begin{array}{c}\text { Streptococcus thermophilus } \\
\text { (DSM20259) }\end{array}$} \\
\hline & \multicolumn{8}{|c|}{ Olive oil \% } \\
\hline & 0.0 & 1.0 & 0.0 & 1.0 & 0.0 & 1.0 & 0.0 & 1.0 \\
\hline 0 & $8.1 \times 10^{7}$ & $8.9 \times 10^{7}$ & $6.6 \times 10^{7}$ & $7.3 \times 10^{7}$ & $8.7 \times 10^{7}$ & $6.0 \times 10^{7}$ & $8.3 \times 10^{7}$ & $7.4 \times 10^{7}$ \\
\hline 12 & $3.0 \times 10^{8}$ & $5.2 \times 10^{7}$ & $7.0 \times 10^{6}$ & $2.0 \times 10^{6}$ & $6.4 \times 10^{9}$ & $7.7 \times 10^{5}$ & $5.7 \times 10^{9}$ & $1.3 \times 10^{6}$ \\
\hline 24 & $4.0 \times 10^{5}$ & & $9.0 \times 10^{7}$ & $6.2 \times 10^{3}$ & $4.3 \times 10^{8}$ & $2.2 \times 10^{2}$ & $4.2 \times 10^{6}$ & $4.4 \times 10^{2}$ \\
\hline 36 & $4.0 \times 10^{2}$ & & $4.0 \times 10^{5}$ & $\underline{-}$ & $5.0 \times 10^{4}$ & & $5.6 \times 10^{4}$ & \\
\hline 48 & - & $\bar{L}$ & $8.0 \times 10^{3}$ & $\overline{ }$ & $2.0 \times 10^{2}$ & & $7.2 \times 10$ & \\
\hline 60 & & & - & & & & & \\
\hline 72 & & & & & & & & \\
\hline
\end{tabular}

TABLE IX: CFU/ML OF BACILLUS CEREUS (ATCC9639) INOCULATED IN BARKI SHEEP CHEESE YOGHURT MADE USING DIFFERENT PROBIOTIC STRAINS AND SUPPLEMENTED WITH OLIVE OIL AND STORED FOR 72 HOURS AT $5{ }^{\circ} \mathrm{C}$

\begin{tabular}{|c|c|c|c|c|c|c|c|c|}
\hline \multirow{2}{*}{$\begin{array}{l}\text { Storage } \\
\text { (hrs) }\end{array}$} & \multicolumn{2}{|c|}{$\begin{array}{c}\text { Bifidobacterium } \\
\text { (ATCC15708) }\end{array}$} & \multicolumn{2}{|c|}{$\begin{array}{l}\text { L. acidophilus } \\
\text { (ATCC4356) }\end{array}$} & \multicolumn{2}{|c|}{$\begin{array}{l}\text { L. bulgaricus } \\
\text { (ATCC7995) }\end{array}$} & \multicolumn{2}{|c|}{$\begin{array}{c}\text { Streptococcus thermophilus } \\
\text { (DSM20259) }\end{array}$} \\
\hline & 0.0 & 1.0 & 0.0 & 1.0 & 0.0 & 1.0 & 0.0 & 1.0 \\
\hline 0 & $7.8 \times 10^{7}$ & $8.14 \times 10^{7}$ & $8.29 \times 10^{7}$ & $9.18 \times 10^{7}$ & $9.29 \times 10^{7}$ & $8.5 \times 10^{7}$ & $6.19 \times 10^{10}$ & $9.8 \times 10^{7}$ \\
\hline 12 & $3.3 \times 10^{10}$ & $6.8 \times 10^{6}$ & $6.7 \times 10^{9}$ & $6.2 \times 10^{6}$ & $6.12 \times 10^{9}$ & $6.6 \times 10^{5}$ & $5.2 \times 10^{8}$ & $9.0 \times 10^{5}$ \\
\hline 24 & $7.7 \times 10^{7}$ & $4.4 \times 10^{3}$ & $4.5 \times 10^{7}$ & $8.8 \times 10^{4}$ & $5.4 \times 10^{8}$ & $8.2 \times 10^{2}$ & $4.9 \times 10^{6}$ & $2.0 \times 10^{2}$ \\
\hline 36 & $9.9 \times 10^{4}$ & & $6.8 \times 10^{5}$ & & $6.0 \times 10^{7}$ & & $5.7 \times 10$ & \\
\hline 48 & $5.2 \times 10^{2}$ & & $3.9 \times 10^{4}$ & & $3.3 \times 10^{3}$ & & & \\
\hline 60 & . & & $2.3 \times 10^{2}$ & & & & & \\
\hline 72 & & & & & & & & \\
\hline
\end{tabular}


TABLE X: CFU/ML OF SALMONELLA TYPHIMURIUM (ATCC25566) INOCULATED IN BARKI SHEEP CHEESE YOGHURT MADE USING DIFFERENT PROBIOTIC STRAINS AND SUPPLEMENTED WITH OLIVE OIL AND STORED FOR 72 HOURS AT $5{ }^{\circ} \mathrm{C}$

\begin{tabular}{|c|c|c|c|c|c|c|c|c|}
\hline \multirow{3}{*}{$\begin{array}{l}\text { Storage } \\
\text { (hrs) }\end{array}$} & \multicolumn{2}{|c|}{$\begin{array}{c}\text { Bifidobacterium } \\
\text { (ATCC15708) }\end{array}$} & \multicolumn{2}{|c|}{$\begin{array}{l}\text { L. acidophilus } \\
\text { (ATCC4356) }\end{array}$} & \multicolumn{2}{|c|}{$\begin{array}{l}\text { L. bulgaricus } \\
\text { (ATCC7995) }\end{array}$} & \multicolumn{2}{|c|}{$\begin{array}{c}\text { Streptococcus } \\
\text { thermophilus } \\
\text { (DSM20259) }\end{array}$} \\
\hline & \multicolumn{8}{|c|}{ Olive oil \% } \\
\hline & 0.0 & 1.0 & 0.0 & 1.0 & 0.0 & 1.0 & 0.0 & 1.0 \\
\hline 0 & $8.16 \times 10^{7}$ & $7.7 \times 10^{7}$ & $8.19 \times 10^{7}$ & $9.9 \times 10^{7}$ & $8.8 \times 10^{7}$ & $7.8 \times 10^{7}$ & $6.8 \times 10^{7}$ & $8.8 \times 10^{7}$ \\
\hline 12 & $9.0 \times 10^{10}$ & $5.0 \times 10^{6}$ & $3.0 \times 10^{6}$ & $6.0 \times 10^{6}$ & $7.4 \times 10^{9}$ & $3.0 \times 10^{5}$ & $9 \times 10^{10}$ & $6.8 \times 10^{3}$ \\
\hline 24 & $5.0 \times 10^{9}$ & $4.4 \times 10^{5}$ & $7.0 \times 10^{7}$ & $7.2 \times 10^{4}$ & $6.6 \times 10^{7}$ & & $8.3 \times 10^{7}$ & \\
\hline 36 & $7.0 \times 10^{6}$ & & $5.2 \times 10^{5}$ & & $8.7 \times 10^{5}$ & & $6.8 \times 10$ & \\
\hline 48 & $2.0 \times 10^{2}$ & & $6.5 \times 10^{2}$ & & $4.4 \times 10^{2}$ & & & \\
\hline 60 & & & & & & & & \\
\hline 72 & & & & & & & & \\
\hline
\end{tabular}

\section{CONCLUSION}

Consumers are more willing to accept natural extracts as preservatives than synthetic ones due to the nature and relative safety of natural products. From the results obtained, it could be concluded that olive oil can be added to fermented milk to increase both the quality and the nutritional value of the final dairy product, without inducing any negative effects on the viability of the lactic acid bacteria. Using olive oil (1\%) had high antibacterial effect against the four tested pathogens. E. coli (ACCT8739) was more sensitive, followed by Salmonella typhimurium (ATCC25566) and the most resistant microorganism was Staphylococcus aureus (ATCC6538). Olive oil $(1 \%)$ had no effect against the growth of different probiotic bacterial strains. The antibacterial activity of cheese yoghurt supplemented with olive oil $(1 \%)$ was higher than control treatment because olive oil supported the growth of Lactic acid bacteria. It is recommended that olive oil (1\%) can be used as a natural and safe anti-microbial substance in Barki cheese yoghurt and other dairy products, and olive oil may well have the beneficial role in promoting probiotic bacteria and inhibiting harmful bacteria.

\section{REFERENCES}

[1] El-Bayoumi M.M., Nutritional and immunological studies on Barki sheep milk Ph.D. Thesis, Alexandria university, Damanhur, Egypt, 2008.

[2] Havel R. J., Milk fat consumption and human health: NIH and other American government regulations. In: Milk composition, production and Biotechnology, R.A.S. Welch D.J.W. Burns, S.R. Davis, A.I. Popay and C.G. Prosser (Edts).CAB International, NY, USA, 1997, pp. 13-22.

[3] Berger Y., Cannas A., Billon P., Mckusick B., Bocquier F., Marnet P., Caja G., and D. Thomas, Principles of sheep dairying in North America, 2004, pp. 3-12.

[4] Gandul-Rojad B., M. R.L. Cepero and M.I. Minguez-Mosquera, Use of chlorophyll and carotenoid pigment composition to determine authenticity of virgin olive oil, J. A M. Oil Chem., 2000.

[5] Ocakoglu D., F. Tokatli, B. Ozen and F. Korel, Distribution of simple phenols, phenolic acids and flavonoids in Turkish monovarietal extra virgin olive oils for two harvest years, Food Chem., 2009, 113:401410.

[6] Guler Z., Changes in salted yoghurt during storage, Int. J. Food Sci. Technol., 2007, 42:235-245.

[7] Senal E., M. Atamar, A. Gursory and F.S. Oztekin, Changes in some properties of strained (Suzme) goat's yoghurt during storage, Small Ruminant Res., 2011, 99:171-177.

[8] Mollet B., I. Rowland, Functional foods: At frontier between food and pharma, Curr. Opin. Biotechnol., 2002, 13:483-485.

[9] EL-Sayed, NH., F.A. H. EL-Senaity, Manufacture of Labneh from goat's milk, J. Agric. Sci. Mansoura Univ., 1993, 18:3310-3316.

[10] Salem M.M.E., Mona A.M. AbdEL-Gawad, Fatma A. M., Hassan B. A. Effat, The use of symbiotic for production of functional low fat Labneh, Pol. J Food Nutr. Sci., 2007, 16:151-159.
[11] Al-Otaibi, M. and H. El-Demerdash, Improvement of the quality and shelf life of concentrated yoghurt (Labneh) by the addition of some essential oils, Afr. J. Microbiol. Res., 2008, 2:156-161.

[12] Mohammed SHS., W.M. Zaky, J.M. Kassem, H.M. Abbas, M.M. E Salem and H.A.H. Said-AlAhl, Impact of antimicrobial properties of some essential oils on cheese yoghurt quality, Word Applied Sci. J., 2013, 27:497-507.

[13] Thabet H.M., Q.A. Nogaim, A.S. Qasha, O. Abdoalaziz and Nalnseme, Evaluation of the effects of some plant derived essential oils on shelf life extension of Labneh, Merit Res. J. Food Sci. Technol., 2014, 2:814.

[14] Ling Guo, Qi Sun, Shaoying Gong, Xue Bi, Wen Jiong, Weixue and Peng Fei, Antimicrobial activity and Action Approach of the olive oil polyphenol Extract Against Listeria monocytogenes. Front. Microbial., 2019. https \doi.org \10.3389\ Fimicd.20.19.01586.

[15] Filomena Nazzaro, Florinda Fratianni, Rosaria Cozzollino, Antonella Martignetti, Livia Malorni, Vincenzo DeFeo, Adriano G, Cruz and Antonio d Acierno, Antibacterial Activity of Three Extra Virgin olive oils of the Campania Region, Southern Italy, Related to their poly phenol content and composition, Microorganisms, 2019, 7(9), 321.

[16] Panesar P.S., Fermented Dairy Products: Starter Cultures and Potential Benefits, Food Nutr. Sci, 2011, 2(1):47-51.

[17] Liu S N, Han. Y, Zhan Zj, Lactic Acid Bacteria in Traditional Fermented Chinse Foods, Food Res. Int., 2010, 44(3):643-651.

[18] Sharma R, Sanodiya BS, Bagrodia D, Pandey M, Sharma A, Bisen P.S., Efficacy and Potential of Lactic Acid Bacteria Modulating Human Health, Int. J. PharmaBio. Sci., 2012, 3(4):935-948.

[19] Steele J, Broadbent J, Kokj, Perspective on the contribution of Lactic Acid Bacteria to Cheese Flavor Development, Curr. Opin. Biotechnol. 2013, 24(2):135-141.

[20] Zanello, G. et. al., Saccharomyces boulardii effects on gastrointestinal diseases, Curr. Issues Mol. Biol., 2009, 11(1)) 47-58.

[21] Boyle R.J., Robins-Browne R.M., Tang M. L.K., Probiotic use in clinical practical: What are the risk?, The American Journal of Clinical Nutrition. 73(6), 2006, 1256-1264.

[22] Delecenserie V.et al., Immunomodulatory effects of probiotics in the intestinal tract, Current Issues in Molecular Biology, 2008, 10(1|2) 37.

[23] Awad H. et al., Comparison between killed and living probiotic usage versus placebo for the prevention of necrotizing enterocolitis and sepsis in neonates, Pak. J. Biol. Sci., 2010, 13(6), 253-262.

[24] Reid. G.et al., Potential uses of probiotics in clinical practice, Clin. Microbiol. Rev., 2003, 16(4) 658-672.

[25] Shan, N.P., Functional Cultures and Health Benefits, Int. Dairy J., 2007, 17(11):1262-1277.

[26] Ali A., Beneficial Role of Lactic Acid Bacteria in Food Preservation and Human Health Res, J. Microbial., 2010, 5(12):1213-1221.

[27] Marhamatizadeh M.H., Karmand, A.R. Farokhi, RRafatjoo and S. Rezade, The effects of malt extract on the increasing growth of probiotic bacteria Lactobacillus acidophilus and Bifidobacterium bifidium in probiotic milk and yoghurt, Journal of food Technology and Nutrition, 2011, 8, pp. 78-84.

[28] Haddadin M.S.Y., Effect of Olive Leaf Extracts on the Growth and Metabolism of Two Probiotic Bacteria of Intestine Origin, Pakistan Journal of Nutrition, 2010, Vol. 9, No. 8, pp. 787-793.

[29] Medina E., DeCastro, Romero and Brenes, Comparison of the Concentration of Phenolic Compound in Olive Oils and Other Plant Oils: Correlation with Antimicrobial Activity, Journal of Agricultural and Food Chemistry, 2006, 54 (14), pp. 4954-4961.

[30] Behrad S., M., Y. Yusof, K.L. Goh and A.S. Baba, Manipulation of Probiotics Fermentation of Yoghurt by Cinnamon and Licorice: Effect on Yoghurt Formation and Inhibition of Helicobacter pylori Growth in Vitro, World Academy of Science, Engineering and Technology, 2009, Vol. 60, pp. 590-594. 
[31] Bea H.C., Lee J.Y., Nam M.S., Effect of red ginseng extract on growth of Lactobacillus sp., Escherichia coli and Listeria monocytogenes in PH controled medium, Koraen J. Food Sci. An., 2005, 25:257-264.

[32] Abbasi M.A., Surrey effect avishan on Bifidobacterium bifidium and Lactobacillus acidophilus probiotic batteries grow in produce of probiotic yoghurt and milk, Doctors of Veterinary Medicine Thesis Islamic Azad University, Number 694, Azad University of Kazeroon, 2009.

[33] Rezazadeh Z., M.H. Marhanamatizadeh, M. Radi, Effect of Vallilin on Lactobacillus acidophilus and Bifidobacterium bifidium and evaluation of its physicochemical and sensory properties in probiotics yoghurt. Journal of Applied Environmental and Biological Sciences, 2015, 2090-4274.

[34] Eduardo M, A. DeCastro, Concepcion R. and Manuel B., Comparison of the Concentrations of Compounds in Olive Oil and Other plant OILS: Correlation with Antimicrobial Activity, J. Agric. Food Chem., 2006, 54, 4954-4961.

[35] Cornu M.C., A. Marchand E. Meurville and J.M Belin, Incidences des composes phenoliques sur des bacteries lactiques et acetiques isoiees du vin, Sci. Aliment, 1984, 4:73-79.

[36] Stead D., The effect of chlorogenic, gallic and quinic acids on the growth of spoilage strains of Lactobacillus collinoides and Lactobacillus brevis, Lett. Appl. Microbial., 1994, 18:112-114.

[37] Vivas N., A. Lonvaud-Funel and Y. Glories, Effect of phenolic acids and anthocyanins on growth, viability and malolactic. 1997, 14:291300.

[38] Reguant C., A. Bordons, L. Arola and Rozes, Influence of phenolic compounds on the physiology of Enococcus centi from wine, J. Appl. Microbial., 2000, 88:1065-1071.

[39] Bergsson G., Arnfinnsson, J., Steinrimsson, O., Thomar, H., In vitro killing of Candida albican by fatty acids and monoglycerides. Antimicrob, Agents Chemother., 2001, 45, 3209-3212.

[40] Trombetta D., Saija A., Bisignano G., Arena S., Caruso S., Mazzanti G., Uccella N., Castelli F., Study on the mechanisms on the antibacterial action of some plant alfa-beta unsaturated aldehydes, Lett. Appl. Microbial, 2002, 35, 285-290.

[41] F Fancello, C. Multineddu, M. Santona, P. Deiana, G. Zara, I. Mannazzu, M. Budroni, S. Dettori and S. Zara, Bacterial Biodiversity of Virgin olive oil and their Potential Biotechnological Exploitation, Microorganism, 2020, 8, 97.

[42] Markin D., Duek L; Bredicevsky I., In vitro antimicrobial activity of olive leaves. Mycoses, 2003, 46, 132-136.

[43] Keceli T. R.; Robibson R.K.; Gordon M.H., The role of olive oil in preservation of yoghurt cheese, Int. J. Dairy Technol., 1999, 52, 68-72.

[44] Puupponen-Pimia R., Nohynek L., Meier C.; Kahkonen M., Heinonen M., Hopia A., Oksman-Caldenty K.M., Antimicrobial properties of phenolic compounds from berries, J. Appl. Microbiol., 2001, 90,494507.

[45] Park B., Kim J., Lee S., Kim K.S., Takeoka G.R., Ahn Y., Kim J., Selestive, growth-inhibiting effects of compounds identified in Tabebuia impetiginosa inner bark on human intestinal bacteria, $J$. Agric. Food Chem., 2005, 53, 1152-1157.

[46] Marcel W L Koo, Chi H Cho, Pharmacological effects of green tea on the gastrointestinal system, Eur. J. Pharmacol. 2004, 500.177-185.

[47] Scalbert A., Monrad Manach C., Remesy C., Absorption and metabolism of polyphenols in the gut and impact on health, Biomed. Pharmacother., 2002, 56, 276-282.

[48] Simon A.L., Renouf M., Hendrich S., Murphy P.A., Human gut microbial degradation of flavonoids: structure-function relationships, J. Agric. Food Chem., 2005, 53, 4258-4263.

[49] Pretty I.A., Gallagher M.J., Matin M.V., Edgar W.M., Higham S.M., A study to assess the effects of a new detergent free, olive oil form nlationdentifrice in vitro and vivo, J. Dent., 2003, 31, 327-332.

[50] Radford S.A., Tassou C.C., Nychas G.J.E.,Board R.G., The influence of different oils on the death rate of Salmonella enteritidis in homemade mayonnaise, Lett Appl. Microbiol., 1991, 12, 125-128.

[51] Alwaili N. S.Clinical and mycological benefits of topical application of honey, olive oil and beeswax in diaper dermatitis. Clinical Microbiology and Infection ,2004,11(2),160-163.

[52] Harish K., Varghese T., Probiotics in humans-evidence based review, Calicut Med. J., 2006, 4(4) 3.

[53] Marhamatizadeh M.H., Effect of Garlic and Dill Extract on Yoghurt Probiotic Bacteria (Bifidobacterium bifidum and Lactobacillus acidophilus) and their role in rat's Triglycerides and Cholesterol. Bulletin of Environment, Pharmacology and Life Sciences, 2015, 4 (3) February:10-15.

[54] Marhamatizadeh M.H., R. Rafatjioo, A.R. Farokhi, M. Karm and S. Rezaazade, The study of soya extract on the growth of probiotic Lactobacillus acidophilus and Bifidobacterium bacteria in probiotic milk and yoghurt, Journal of Veterinary Pathobiology, 2009, 1, pp. 2328.

[55] Rybalchenko, O.V., Boomdarenko, V.M., Verbitskaia, N.B., Antagonistic effect of bacteriocinogenic Lactobacillus acidophilus on Klebsiella pneumonia, Citrobacter freundii and Proteus mirabilis ceels, Zhurnal Mikrobiologii, Epidemiologii, Immunobiologii, 2006 , (7) $8-11$.

[56] Szajewska H., Mrukowicz J.Z., Probiotics in the treatment and prevention of acute infectious diarrhea infants and children: a systematic review of published randomized, double- blind, placebocontrolled trials, Journal of Pediatric Gastroenterology and Nutrition, 2001, 33(2) S17-S25. 